\title{
Centro de Estudos Sociais, Faculdade de Economia da
}

\section{Universidade de Coimbra}

Joāo Arriscado Nunes

Citer ce document / Cite this document :

Arriscado Nunes Joāo. Centro de Estudos Sociais, Faculdade de Economia da Universidade de Coimbra. In: Recherches en anthropologie au Portugal, $n^{\circ} 1,1992$. pp. 61-64;

doi : 10.3406/rap.1992.937

http://www.persee.fr/doc/rap_1240-3474_1992_num_4_1_937

Document généré le 25/04/2016 
Centro de Estudos Sociais". Faculdade de Economia da Universidade de Coimbra.

Le Centro de Estudos Sociais est une association scientifique à but non lucratif. créée en 1978 par un groupe d'enseignants de la Faculté des Sciences Economiques de l'Université de Coimbra. Le CES compte, parmi sa quarantaine de chercheurs permanents ou associés. des sociologues. des économistes, des géographes. des spécialistes en littérature et études culturelles, des juristes et des médecins, liés ou non à l'Université. La recherche menée au CES est interdisciplinaire et intégrée, portant sur les terrains des différentes sciences sociales et humaines, et développant des perspectives et des outils théoriques, analytiques et méthodologiques qui se veulent nouveaux et innovateurs. L'activité du CES a porté, surtout, sur la connaissance de la société portugaise - une société dont le niveau de développement est moyen et qui connait un processus de transformation rapide -, à partir d'une perspective qui tient compte de l'internationalisation et de la globalisation des rapports sociaux en cours.

Les objectifs prioritaires du CES sont :

- la promotion de la recherche scientifique sur la société portugaise ;

- la réalisation d'études comparatives, notamment sur le Portugal, le Brésil et les pays Africains de langue portugaise :

- la coopération avec d'autres institutions scientifiques, au Portugal et dans d'autres pays, et la participation à des réseaux internationaux de recherche comparative :

- la prestation de services à la communauté, et autres activités de prestation de services :

- la création de banques de données et d'autres infrastructures de soutien et d aide à la recherche :

- la diffusion des études et recherches menées au CES à travers la publication de la Revista Critica de Ciências Sociats. de la série de documents de travail Oficina do CES et d'autres publications.

Le CES est organisé en cinq Groupes de Recherches : Études Sociales de la Médecine et de la Santé : Études Culturelles Comparées: Culture. Tourisme et Société ; Études sur la Société, le Droit et l'Administration : Études sur les Systèmes Productifs et l’Internationalisation.

Dossier fourni par João Arriscado Nunes. enscignant de Sciences Sociales à la Faculdade de Economia. Universidade de Coimbra. à qui nous adressons nos remerciements 
Plusieurs projets de recherche sont en cours ou ont déjà été conclus par les différents groupes de recherche. ou par des chercheurs individuels:

- L'État, l'économie et la reproduction sociale dans la semipériphérie du système-monde (responsable : Boaventura de Sousa Santos).

- Justice et communauté à Macao (responsable : Boaventura de Sousa Santos).

- Structures socio-économiques et marché du travail (responsable: Claudino Ferreira).

- Automation des bureaux et qualification professionnelle (responsable : Virginia Ferreira).

- Un marché d'idées pour l'emploi dans la région du Baixo Mondego (responsable : José Reis).

- Les réseaux de la famille: la construction interactionnelle des solidarités primaires (responsable : João Arriscado Nunes).

- Les nouvelles technologies de l'information, la formation des employées de bureau et l'emploi (responsable : Virgínia Ferreira).

- Structures d’entreprise et spécialisation dans la région de Leiria (responsable : José Reis).

- Coopérativisme et droit de la coopération (responsable: Rui Namorado).

- Formes de médiation et concertation sociales au Portugal (responsable : António Casimiro Ferreira)

- L'administration de la justice au Portugal (responsable : Boaventura de Sousa Santos).

- Education pour tous (responsable : Lucilia Salgado).

- Politiques sociales comparées - Portugal et Brésil (responsables: Boaventura de Sousa Santos et Pedro Hespanha (Portugal), Amélia Cohn et Aldaíza Sposati (Brésil).

- Tourisme et culture au Portugal (responsable : Carlos Fortuna).

- Le local et la politique au Portugal (responsable : Fernando Ruivo).

- L'articulation des soins primaires et des soins à l'hôpital (responsable : Graça Carapinheiro).

- Le patrimoine commun de l'humanité - vers un droit international de la solidarité ? (responsable : José Manuel Pureza).

- Internationalisation et modernisation de l'industrie; le "secteur internationalisé » et les «régimes technologiques " (responsable : José Reis).

- La taverne, le boire, ses saveurs et ses savoirs (responsable : Pedro de Andrade). 
- Les formes juridiques de coopération entre firmes (responsable : Maria Manuel Leitão Marques).

- État et société au Portugal : État. politiques publiques et régulation socio-économique. Observatoire social permanent inter-centres de l'économie et de la société portugaises (responsable: Boaventura de Sousa Santos).

- Intellectuels, culture et société : quatre études sociologiques (responsable : Maria Irene Ramalho).

- Émigration et identité (responsable : Graça Capinha).

- Matrices de qualité dans la prestation de services (responsable: Virgínia Ferreira).

- Mobilité spatiale et transformations des identités: une étude comparative des processus de transformation chez des migrants internationaux (responsable: Bela Feldman-Bianco et Pedro Hespanha).

- Classes sociales et trajectoires de classe au Portugal - projet international (responsable : Erik Olin Wright).

- Recherches transnationales en études socio-juridiques (responsables: Boaventura de Sousa Santos (Coimbra), David Trubek (Madison), Hazel Genn (Londres), Richard Abel (Los Angeles). William Felstiner (Santa Barbara).

- Discours économique et controverses publiques (responsable : João Arriscado Nunes).

Le CES diffuse des "progress reports " et les résultats de ces projets de recherche, ainsi que la collaboration d'autres chercheurs, à travers ses publications :

- la Revista Crítica de Ciências Sociais, dont 37 numéros ont été publiés depuis 1978 ;

- la série de documents de travail Oficina do CES ;

- la collection Saber Imaginar o Social, dirigée par Boaventura de Sousa Santos et publiée par les Edições Afrontamento (Porto).

- des documents audiovisuels, dont le documentaire vidéo Saudade réalisé par Bela Feldman-Bianco, professeur d'anthropologie à UNICAMP (Brésil), en collaboration avec Michael Majoros et Peter O`Neill -. sur l'expérience et la culture des communautés d'immigrés portugais à New Bedford, Massachussetts (USA).

Le CES organise, régulièrement. des séminaires avec la participation des chercheurs du Centre et de chercheurs invités, portugais et étrangers. 
Depuis 1981, le CES a promu plusieurs colloques et cours :

- Premier colloque d’Études Rurales, sur la petite agriculture au Portugal (mars 1981).

- Theorie de l'Histoire. Histoire Sociale, Histoire du Droit (mai 1983).

- Portugal 1974-1984: dix ans de transformations sociales (décembre 1984).

- Les nouvelles frontières de la légalité (en collaboration avec la European Conference of Critical Legal Studies) (avril 1985).

- Espace et industrialisation (mai 1987).

- Médecine et santé au Portugal (septembre 1987).

- Les carrefours du pouvoir local (juillet 1989).

- Premier Congrès Luso-Afro-Brésilien de Sciences Sociales (juillet 1990).

- Le droit et les rapports entre l'économique et le social (novembre 1990).

- Cours de formation en orientation professionnelle sur «La société portugaise: problèmes de modernisation et de transformation » (fëvrier-juillet 1988).

- Cours monographique sur "Familles : conflits et changements», pour professionnels de santé, organisé par le Groupe d'Études Sociales de la Médecine et de la Santé.

Adresse : Centro de Estudos Sociais

Universidade de Coimbra

Colégio S.Jerónimo

Praça D. Dinis

3000 Coimbra - Portugal

Tél. (039) 26459/20354

Fax 351-39-29076

Correspondance : Centro de Estudos Sociais Apartado 3087

3000 Coimbra - Portugal 